\title{
Probing the ionized ISM of the CSS quasar 3C 277.1
}

\section{Observations}

\author{
W. D. Cotton ${ }^{1}$, C. Fanti ${ }^{2,3}$, R. Fanti ${ }^{2,3}$, G. Bicknell ${ }^{4}$, and R. E. Spencer ${ }^{5}$ \\ 1 National Radio Astronomy Observatory ${ }^{\star}, 520$ Edgemont Road, Charlottesville, VA 22903-2475, USA \\ e-mail: bcotton@nrao.edu \\ 2 Istituto di Radioastronomia - INAF, via P. Gobetti 101, 40129 Bologna, Italy \\ 3 Dipartimento di Fisica, Universitá di Bologna, via Irnerio 46, 40126 Bologna, Italy \\ 4 Australian National University, Mt. Stromlo Observatory, Cotter Rd., Weston, ACT 2611, Australia \\ 5 NRAL-Jodrell Bank, University of Manchester, Macclesfield Cheshire, SK11 9DL, UK
}

Received 11 October 2005 / Accepted 3 November 2005

\section{ABSTRACT}

We present new multi-wavelength high resolution VLA polarimetric images of the CSS quasar 3C 277.1 and analyze them with older Merlin and HST [OIII] images. These observations were made to test the hypothesis that the [OIII] emission as well as the long wavelength radio opacity arise in shocks that the quasar jet induces in the ISM. The multi-wavelength radio polarimetric images allow the study of the intervening magnetized plasma using Faraday effects. The HST [OIII] image potentially reveals regions of shock that have cooled sufficiently to radiate. We find no compelling evidence that the jet is directly responsible for the majority of the [OIII] emission or that there is significant dense shocked material in the immediate vicinity of most of the jet. There is evidence for jet-induced shocks on the northern edge of the eastern lobe. We find a spectral age of the radio source of $\sim 4 \times 10^{4} \mathrm{yr}$ giving advance speeds of the lobes of $0.15 \mathrm{c}$ and $0.06 \mathrm{c}$ for the western and eastern lobes. The strong Faraday effects appear to be confined within the inner $3 \mathrm{kpc}$ as does the [OIII] emission.

Key words. polarization - galaxies: quasars: individual: 3C 277.1 - galaxies: jets - galaxies: nuclei - radio continuum: galaxies

\section{Introduction}

Compact Steep Spectrum (CSS) sources (Kapahi 1981; Peacock \& Wall 1982) are powerful extragalactic radio sources with sub-galactic apparent linear dimensions and steep spectra $\left(\alpha \leq-0.5, S \propto v^{\alpha}\right)$. The angular dimensions of these sources are of the order of a few arc-seconds and they are related as a class to the more compact Giga-hertz Peaked Sources (GPS). Fanti et al. (1995) and Readhead et al. (1996) suggest that GPS and CSS sources represent part of the growth sequence leading to the large scale double radio sources. A detailed review is given by O'Dea (1998).

O'Dea (1998) has shown a tight inverse correlation between the frequency of the spectral peak in CSS and GPS sources with linear size. This correlation could be the result of synchrotron self absorption opacity (O'Dea 1998). Alternately, Bicknell et al. (1997) and Bicknell et al. (2003) have suggested that the opacity could be due to free-free absorption from shock ionized gas in the ISM and this effect can also reproduce the spectral peak-linear size relationship.

* The National Radio Astronomy Observatory (NRAO) is operated by Associated Universities Inc., under cooperative agreement with the National Science Foundation.
Observations of emission lines by Gelderman \& Whittle (1994) have shown that CSS sources have a denser than normal for AGNs ionized component in the ISM and suggest this is the result of jet-ISM interactions. This is further supported by the HST observations of Axon et al. (2000) of a number of CSS sources. Most of the sources observed had extended [OIII] emission line gas that appears related to the radio emission.

Further evidence for interaction of CSS jets with interstellar gas comes from the polarization measurements of Saikia \& Gupta (2003) where CSS appear to be more asymmetric in polarization properties than larger radio sources. They suggest that the asymmetry is caused by interaction of the jets with in-falling gas which eventually fuels the central engine. The effects of interaction asymmetries on the arm-length ratios of CSS are also discussed in Jeyakumar et al. (2005). This emission line gas would be expected to provide the free-free opacity required in the Bicknell et al. (2003) model.

Cotton et al. (2003b) have shown that at low frequencies, the inner $3 \mathrm{kpc}$ of CSS sources are strongly depolarized. This depolarization is presumably by the same gas that produces the line emission. Optical images of line emission are only available with sufficient resolution from very few of these sources 
so it is not clear how strongly the ionized gas is associated with the radio jets. Axon et al. (2000) only imaged 11 CSS sources so the optical properties on the size scale of interest here are poorly known.

The Bicknell et al. (2003) shock ionization model predicts that the thermal gas would be very filamentary with a low spatial filling factor. One way to test this hypothesis is through Faraday rotation and depolarization measurements of the radio source as seen through this medium. The denser filaments would have a very high and variable Faraday depth, and would largely depolarize any radio emission passing through them. Emission passing through the holes in the Faraday screen could suffer far less Faraday rotation and depolarization. Thus, the prediction is that the portion of the radio source seen through this screen will be largely depolarized, depending on the blocking factor of the dense Faraday screen, but with only modest (few $100 \mathrm{rad} / \mathrm{m}^{2}$ ) rotation measure. The depolarization should also not increase as rapidly with increasing wavelength as is the case for a more homogeneous screen. Recent observations of the CSS quasar 3C 138 (Cotton et al. 2003a), suggest that the polarized emission from a moving component near the core is largely seen through holes in a dense Faraday screen.

The only suitable candidate for such a test with HST observations is $3 \mathrm{C} 277.1(\mathrm{~J} 1252+5634)$. This is a $1.8^{\prime \prime}\left(8.3 \mathrm{kpc}^{1}\right)$ radio source (Lüdke et al. 1998) in a quasar at $z=0.32$. In the following we discuss new high resolution VLA+VLBA Pietown observations of 3C 277.1 at 3.6, 2 and $1.3 \mathrm{~cm}$ wavelength and analyze them together with the Merlin $6 \mathrm{~cm}$ data of Lüdke et al. (1998) and the HST O[III] image of Axon et al. (2000). In a subsequent paper (Paper II) we will present the results of detailed modeling of this object.

\section{Observations and data reduction}

3C 277.1 was observed using the NRAO VLA array on 7 September 2003 in the "A" configuration also using the VLBA antenna at Pietown, NM, USA. This gives baselines up to $70 \mathrm{~km}$. in length and a reasonable uv coverage due to the northerly declination of $3 \mathrm{C} 277.1$; the J2000 position of the core of $3 \mathrm{C} 277.1$ is $\mathrm{RA}=125226.36$, Dec $=+563419.6$. Observations were made over $12 \mathrm{~h}$, alternating among wavelengths of $3.6,2$, and $1.3 \mathrm{~cm}$. All data processing described here was done in the NRAO AIPS package. The Merlin $6 \mathrm{~cm}$ data of Lüdke et al. (1998) were also reanalyzed.

\subsection{Merlin $6 \mathrm{~cm}$}

The observations were made during $14 \mathrm{~h}$ on 18 April 1995 with a single $15 \mathrm{MHz}$ band-pass centered at $4.994 \mathrm{GHz}$ in both RCP and LCP. These data were calibrated as described in Lüdke et al. (1998). After self calibration, images were made in Stokes $I, Q$, and $U$ using $0.04^{\prime \prime}$ resolution. For subsequent analysis this image was convolved to $0.06^{\prime \prime}$ and $0.12^{\prime \prime}$ resolutions. Polarization amplitudes were bias corrected (AIPS

\footnotetext{
1 We adopt a cosmology with $H_{0}=71, \Omega_{\text {matter }}=0.27 \Omega_{\Lambda}=0.73$ in which at $z=0.32,1^{\prime \prime}=4.62 \mathrm{kpc}$.
}

task POLCO) before forming the fractional polarization. The Merlin $6 \mathrm{~cm}$ results are shown in Fig. 1.

\subsection{VLA $3.6 \mathrm{~cm}$}

The observations were made using two $50 \mathrm{MHz}$ band-passes centered at 8.4351 and $8.4851 \mathrm{GHz}$ in both RCP and LCP. The data were obtained and reduced in the standard mode using $\mathrm{J} 1302+578$ as the astrometric and instrumental polarization calibrator and $\mathrm{J} 1331+305$ (3C 286) as the photometric and polarization angle calibrator. The assumed orientation of the electric vector of the linear polarization of $\mathrm{J} 1331+305$ was $33^{\circ}$ and its flux density was $5.18 \mathrm{Jy}$. After external calibration, several iterations of phase only self-calibration were followed by a single amplitude and phase self calibration. After self-calibration, images were made in Stokes $I, Q$, and $U$ using 0.120" resolution. Polarization amplitudes were bias corrected before forming the fractional polarization. The $3.6 \mathrm{~cm}$ VLA results are shown in Fig. 2.

\subsection{VLA $2 \mathrm{~cm}$}

The observations were made using two $50 \mathrm{MHz}$ band-passes centered at 14.9649 and $14.9149 \mathrm{GHz}$ in both RCP and LCP. $\mathrm{J} 1302+578$ was used as the astrometric and instrumental polarization calibrator and $\mathrm{J} 1331+305$ as the photometric and polarization angle calibrator. The assumed orientation of the electric vector of the linear polarization of J1331+305 was $33^{\circ}$ and its flux density was $3.42 \mathrm{Jy}$. Antenna pointing errors were measured and corrected every $1-1.5 \mathrm{~h}$ using measurements of $\mathrm{J} 1302+578$ at $3.6 \mathrm{~cm}$. Observations of $\mathrm{J} 1331+305$ were calibrated using a standard model of the source. Measurements of 3C 277.1 were immediately preceded and followed by observations of the astrometric phase calibrator with 12 min duration observations of $3 \mathrm{C} 277.1$. A correction was determined for the coherence loss in the phase reference calibrator due to atmospheric phase fluctuations by comparing the ratio of the total flux density in a derived image of J1331+305 with and without self calibration. After external calibration of 3C 277.1, several iterations of phase only self-calibration were made. After self-calibration, images were made in Stokes $I, Q$, and $U$ using $0.06^{\prime \prime}$ resolution. Polarization amplitudes were bias corrected before forming the fractional polarization. The $2 \mathrm{~cm}$ VLA results are shown in Fig. 3.

\subsection{VLA $1.3 \mathrm{~cm}$}

The observations were made using two $50 \mathrm{MHz}$ band-passes centered at 22.4851 and $22.4351 \mathrm{GHz}$ in both RCP and LCP. The observations and reduction were done in the same manner as the $2 \mathrm{~cm}$ observations. The assumed orientation of the electric vector of the linear polarization of $\mathrm{J} 1331+305$ was $33^{\circ}$ and its flux density was $2.50 \mathrm{Jy}$. After self-calibration, images were made in Stokes $I, Q$, and $U$ using $0.06^{\prime \prime}$ resolution. Polarization amplitudes were bias corrected before forming the fractional polarization. The $1.3 \mathrm{~cm}$ VLA results are shown in Fig. 4. 

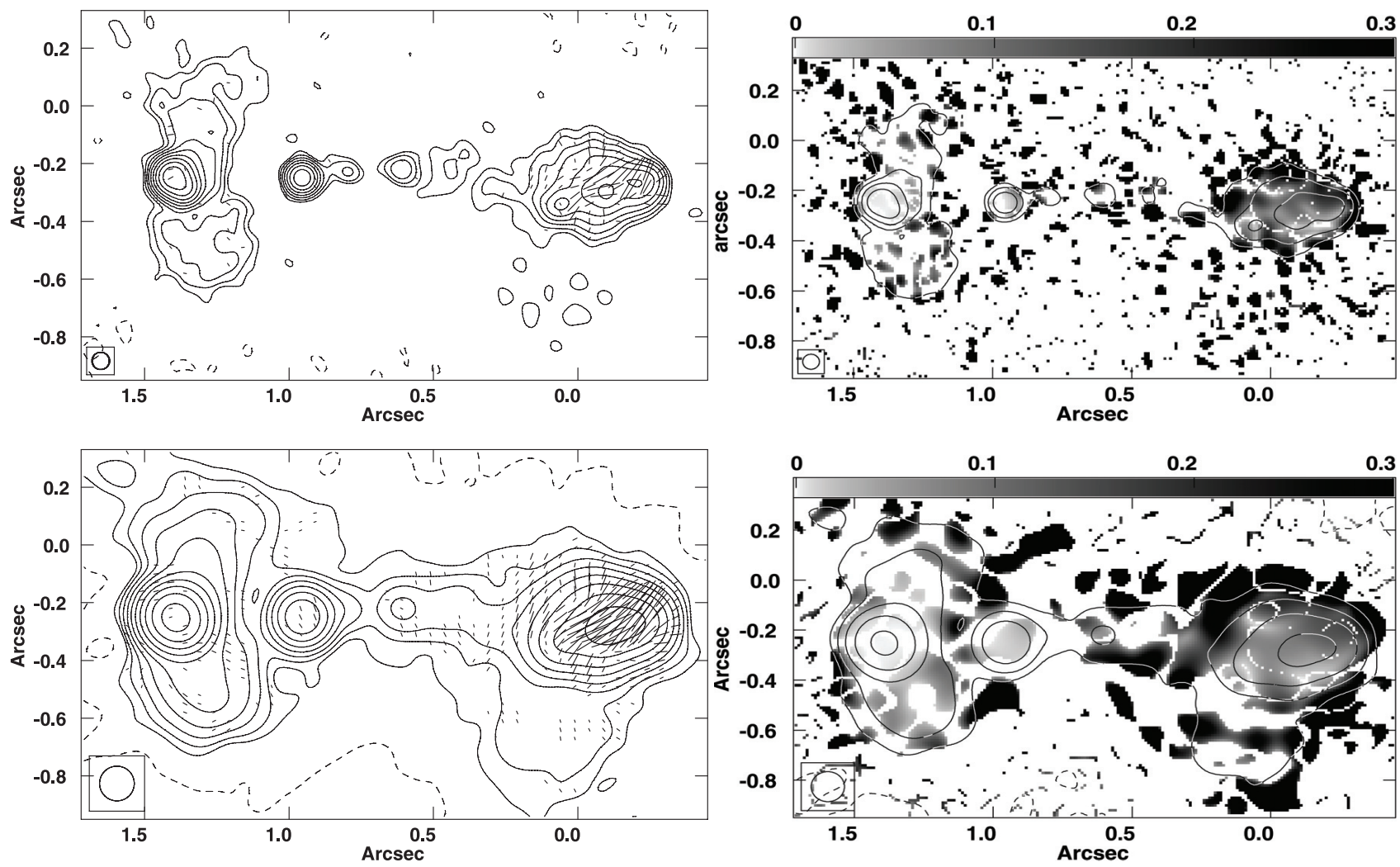

Fig. 1. Upper left: Merlin image of 3C 277.1 at $6 \mathrm{~cm}$ and $0.06^{\prime \prime}$ resolution, contours are total intensity, vectors have lengths proportional to the bias corrected polarized intensity and the orientation of the $E$-vectors. Contours are powers of 2 times $200 \mu \mathrm{Jy}$, negative contours are dashed. The circle in the lower left corner gives the resolution of the image. The image is rotated on the sky by $-42^{\circ}$. Upper right: fractional polarization gray-scale with superposed total intensity contours. fractional polarization scale is shown in the wedge on the top. Contours are powers of 4 times $200 \mu \mathrm{Jy}$. Lower: like upper but at $0.12^{\prime \prime}$ resolution.
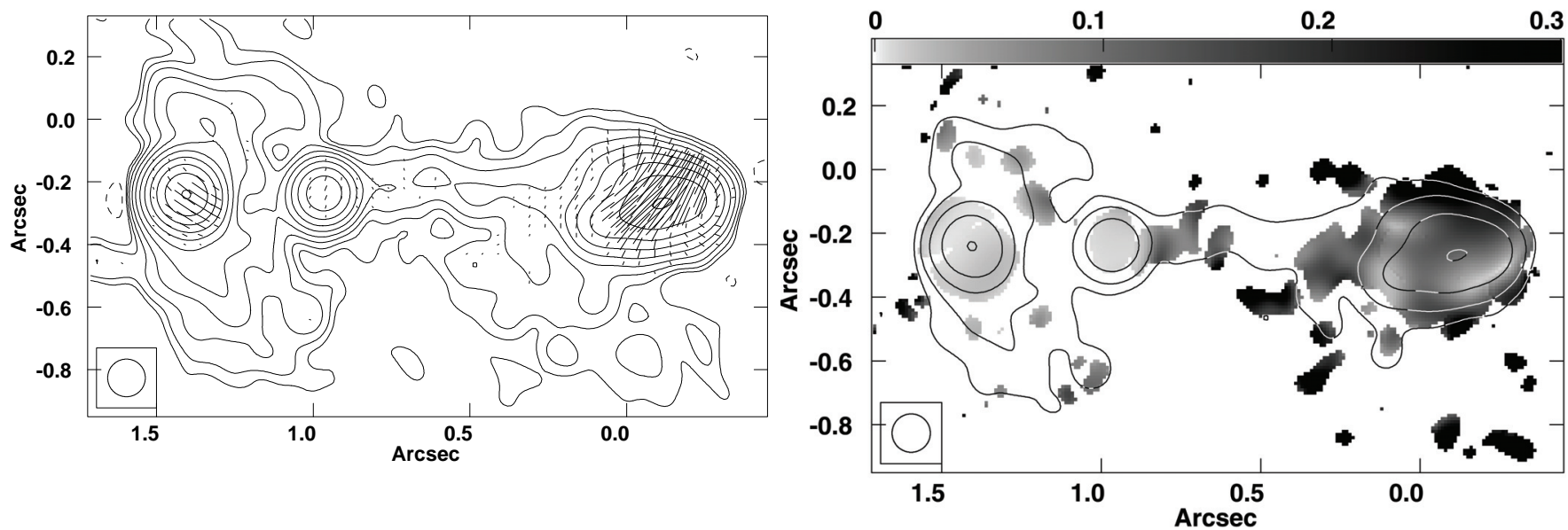

Fig. 2. Left: VLA image of 3C 277.1 at $3.6 \mathrm{~cm}$ and $0.12^{\prime \prime}$ resolution, contours are total intensity, vectors have lengths proportional to the bias corrected polarized intensity and the orientation of the $E$-vectors. Contours are powers of 2 times $100 \mu \mathrm{Jy}$, negative contours are dashed. The circle in the lower left corner gives the resolution of the image. The image is rotated by $-42^{\circ}$. Right: fractional polarization gray-scale with superposed total intensity contours. Fractional polarization scale is shown in the wedge on the top. Contours are powers of 4 times $100 \mu \mathrm{Jy}$.

\subsection{Image alignment}

Two sets of images were made, at $0.12^{\prime \prime}$ for all wavelengths and at $0.06^{\prime \prime}$ for 6,2 and $1.3 \mathrm{~cm}$. In the latter cases, the $0.06^{\prime \prime}$ image was convolved to obtain a resolution of $0.12^{\prime \prime}$. The core is strong and well defined in all images and was used for the fine alignment. All images were then re-sampled onto the same grid for subsequent analysis.

\section{Results}

The rms off-source noise levels in the images given in Figs. 1-4 are summarized in Table 1 . The total intensity (I) and fractional polarization (FPol) at $0.12^{\prime \prime}$ resolution is given in Table 2 for selected positions: the peak of the east lobe, the core and the peak of the west lobe. 

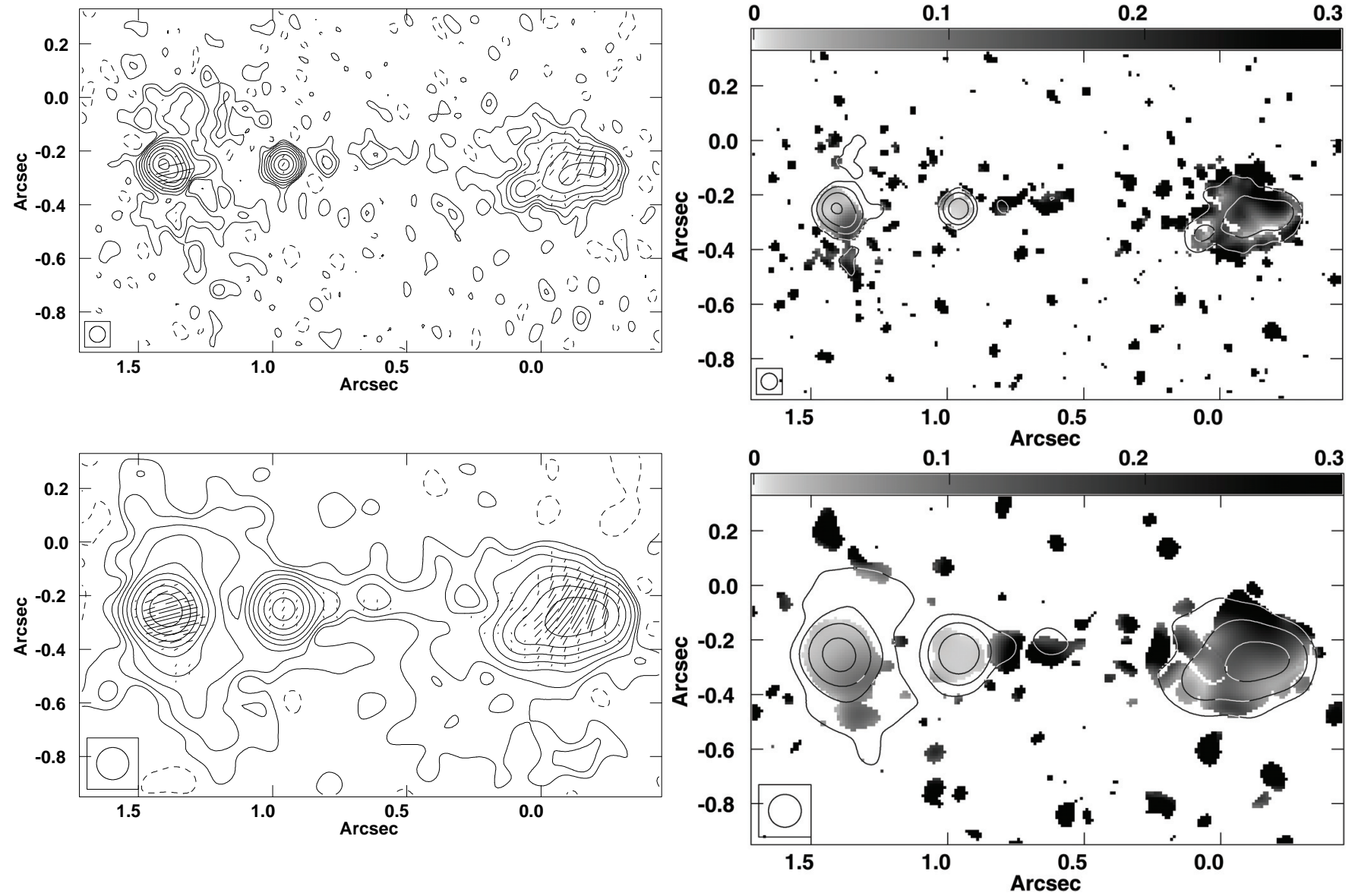

Fig. 3. Upper: VLA image of $3 \mathrm{C} 277.1$ at $2 \mathrm{~cm}$ and $0.06^{\prime \prime}$ resolution, contours are total intensity, vectors have lengths proportional to the bias corrected polarized intensity and the orientation of the $E$-vectors. Contours are powers of 2 times $100 \mu \mathrm{Jy}$, negative contours are dashed. The circle in the lower left corner gives the resolution of the image. The image is rotated by $-42^{\circ}$. Upper right: fractional polarization gray-scale with superposed total intensity contours. Fractional polarization scale is shown in the wedge on the top. Contours are powers of 4 times $100 \mu \mathrm{Jy}$. Lower: like upper but at $0.12^{\prime \prime}$ resolution.

\subsection{Faraday rotation}

Faraday rotation images were derived using a nonstandard AIPS program, ROTM, which determined rotation measures by fitting a $\lambda^{2}$ law using a least squares procedure and weights determined from the SNR of each measurement. Ambiguities in the rotation measure at each pixel were resolved using the closely spaced measurements in $\lambda^{2}$ to predict the phase of measurements with larger $\lambda^{2}$. This fitting procedure was used to derive the rotation measure and the intrinsic polarization angle in each pixel with sufficient signal. The data presented here has a good $\lambda^{2}$ distribution; the shorter wavelengths are sufficiently closely spaced that there are no phase ambiguities and these data easily predict ambiguities at $6 \mathrm{~cm}$. The derived Faraday rotation image is shown in Fig. 5. Any Faraday rotation in the vicinity of the source needs to be corrected by $(1+z)^{2}$, or 1.74 for 3C 277.1 to correct for the difference in emitted and observed wavelengths. The derived intrinsic polarization vectors are shown in Fig. 6.

\subsection{Depolarization}

Since Faraday effects increase with the square of the wavelength, images at longer wavelengths can be depolarized relative to those at shorter wavelengths. The ratios of the fractional polarization at $6,3.6$ and $2 \mathrm{~cm}$ are shown relative to $1.3 \mathrm{~cm}$ in Fig. 7.

\subsection{Spectral index}

The derived spectral index between 6 and $1.3 \mathrm{~cm}$ is shown in Fig. 8. The core of 3C 277.1 has a flat spectral index of -0.1 , and the lobes have steep, power law, spectra with indices of -0.8 to $-1.0^{2}$. The observed wavelengths of all images reported on here are too short be effected by free-free absorption for any reasonable value of electron density.

\subsection{Faraday dispersion}

The work by Burn (1966) and Tribble (1991) have attempted to explain the depolarization with wavelength seen in many sources in terms of a variable Faraday screen within or in front of the source. This variable screen consists of cells with different Faraday properties. The Burn model considers the case where all the cells are the same size and are either much smaller or larger that the resolution of the observations. In the latter

\footnotetext{
${ }^{2}$ Spectral index $\alpha$ is defined as $S_{v} \propto v^{\alpha}$.
} 

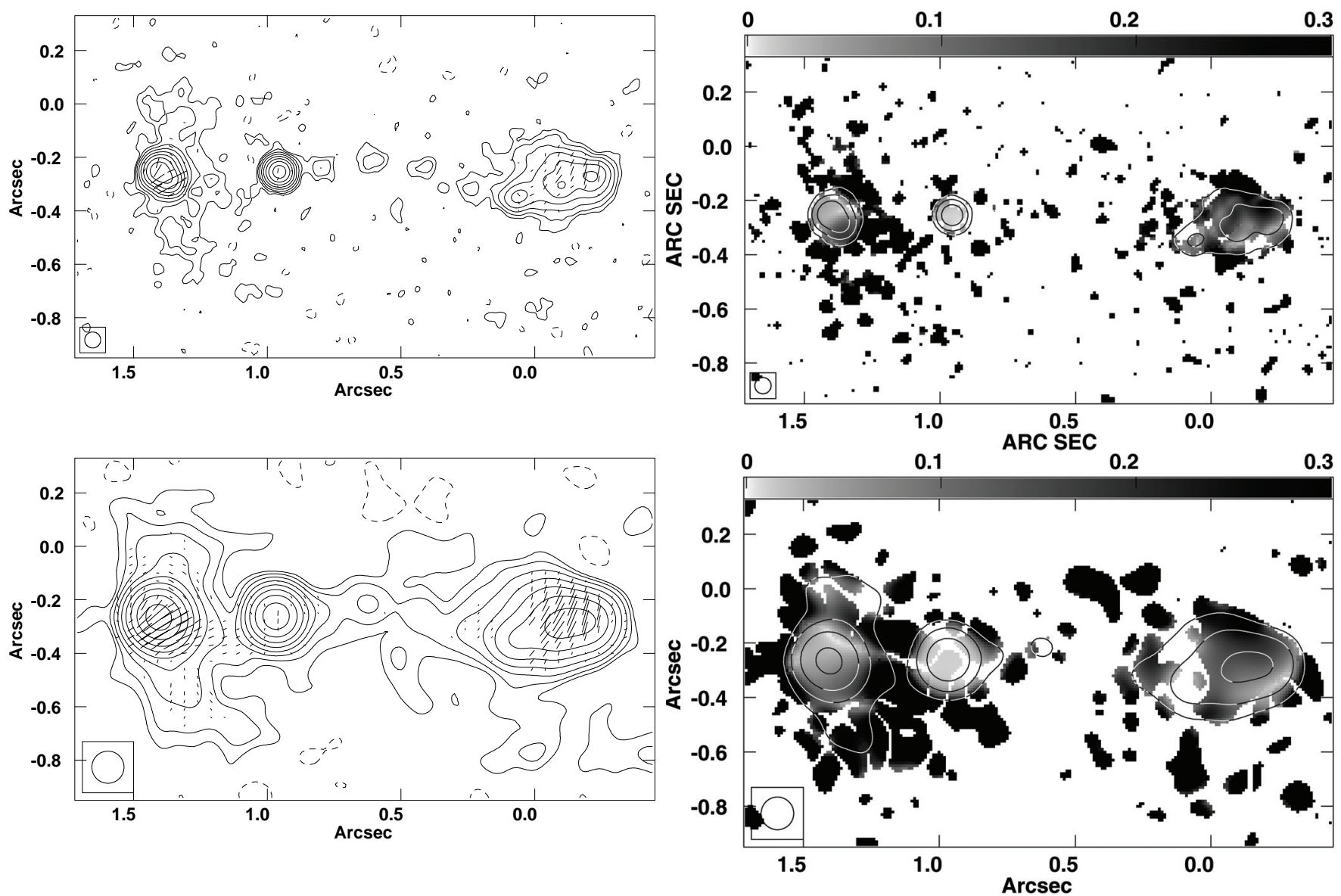

Fig. 4. Upper Left: VLA image of 3C 277.1 at $1.3 \mathrm{~cm}$ and $0.06^{\prime \prime}$ resolution, contours are total intensity, vectors have lengths proportional to the bias corrected polarized intensity and the orientation of the $E$-vectors. Contours are powers of 2 times $100 \mu \mathrm{Jy}$, negative contours are dashed. The circle in the lower left corner gives the resolution of the image. The image is rotated by $-42^{\circ}$. Upper right: fractional polarization gray-scale with superposed total intensity contours. Fractional polarization scale is shown in the wedge on the top. Contours are powers of 4 times $100 \mu \mathrm{Jy}$. Lower: like upper but at $0.12^{\prime \prime}$ resolution.

Table 1. rms noise in images.

\begin{tabular}{lrrrrrr}
\hline \hline & & $0.06^{\prime \prime}$ & & & $0.12^{\prime \prime}$ & \\
$\lambda$ & $I$ & $Q$ & $U$ & $I$ & $Q$ & $U$ \\
$\mathrm{~cm}$ & $\mu \mathrm{Jy}$ & $\mu \mathrm{Jy}$ & $\mu \mathrm{Jy}$ & $\mu \mathrm{Jy}$ & $\mu \mathrm{Jy}$ & $\mu \mathrm{Jy}$ \\
\hline 6 & 170 & 130 & 140 & 450 & 160 & 150 \\
3.6 & & & & 91 & 45 & 48 \\
2 & 110 & 51 & 51 & 130 & 48 & 49 \\
1.3 & 86 & 65 & 65 & 119 & 73 & 72 \\
\hline
\end{tabular}

Table 2. Selected derived values.

\begin{tabular}{lrrrrrr}
\hline \hline & East & \multicolumn{3}{r}{ Core } & West \\
$\lambda$ & $I$ & FPol & $I$ & FPol & $I$ & FPol \\
$\mathrm{cm}$ & $\mathrm{mJy}$ & & $\mathrm{mJy}$ & & $\mathrm{mJy}$ & \\
\hline 6 & 290 & 0.0008 & 49 & 0.0091 & 75 & 0.061 \\
3.6 & 200 & 0.011 & 44 & 0.0081 & 49 & 0.080 \\
2 & 91 & 0.038 & 36 & 0.012 & 21 & 0.11 \\
1.3 & 73 & 0.04 & 39 & 0.011 & 17 & 0.12 \\
\hline
\end{tabular}

case, the result is largely Faraday rotation and only in the former one is depolarization important. In the case of unresolved cells and with the assumption that the rotation measure is randomly distributed with a dispersion $\sigma$, the fractional polarization $(m)$ as a function of $\lambda$ is:

$m(\lambda)=m_{0} \mathrm{e}^{-2 \sigma^{2} \lambda^{4}}$

The Tribble (1991) model extends the Burn analysis by allowing a distribution of cell sizes as well as the Faraday rotation of the cells. In this case:

$$
\left\langle|m(\lambda)|^{2}\right\rangle=m_{0}^{2}\left[\frac{1-\mathrm{e}^{-\left(s_{0}^{2} / 2 b^{2}+4 \sigma^{2} \lambda^{4}\right)}}{1+8 \sigma^{2} \lambda^{4} b^{2} / s_{0}^{2}}+\mathrm{e}^{-\left(s_{0}^{2} / 2 b^{2}+4 \sigma^{2} \lambda^{4}\right)}\right]
$$

where $s_{0}$ is a characteristic scale of the largest cells and $b$ is the size of the beam. However, when $4 \sigma^{2} \lambda^{4}$ is very large this reduces to:

$m(\lambda) \approx m_{0}\left[\frac{s_{0} / b}{2 \sigma \lambda^{2} \sqrt{2}}\right]$.

In this regime is is not possible to separate $s_{0} / b$ and $\sigma$.

The integrated properties of a complete sample of CSS sources are compared to these models by Fanti et al. (2004). In the results given above for 3C 277.1 it is possible to do an analysis on a pixel-by-pixel basis comparing the model to the observations. Unfortunately, for most of our data, the Tribble 


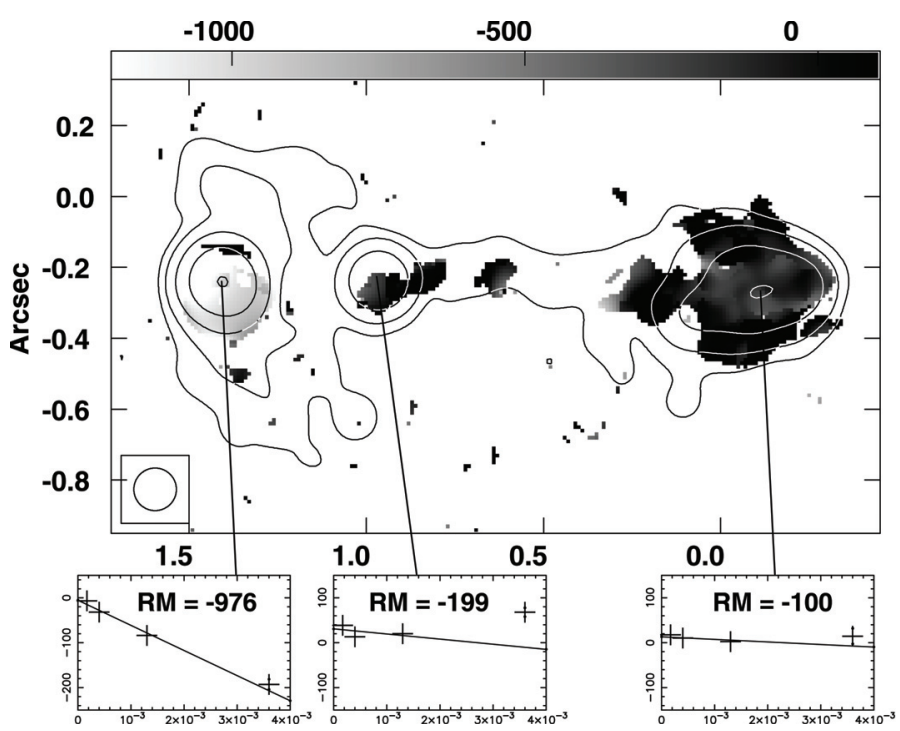

Fig. 5. The gray scale shows the rotation measure image at $0.12^{\prime \prime}$ resolution with superposed contours of the VLA $3.6 \mathrm{~cm}$ image. Rotation measures are in the observer's frame. Plots of the polarization angle as a function of wavelength squared are shown with lines representing the fitted rotation measure for a number of locations. The estimated errors are either given by the vertical size of the cross or are smaller. The bar at the top gives the values of the gray scale in $\mathrm{rad} \mathrm{m}^{-2}$. Contours are powers of 4 times $200 \mu \mathrm{Jy}$. The circle in the lower left corner gives the resolution. The image is rotated by $-42^{\circ}$.

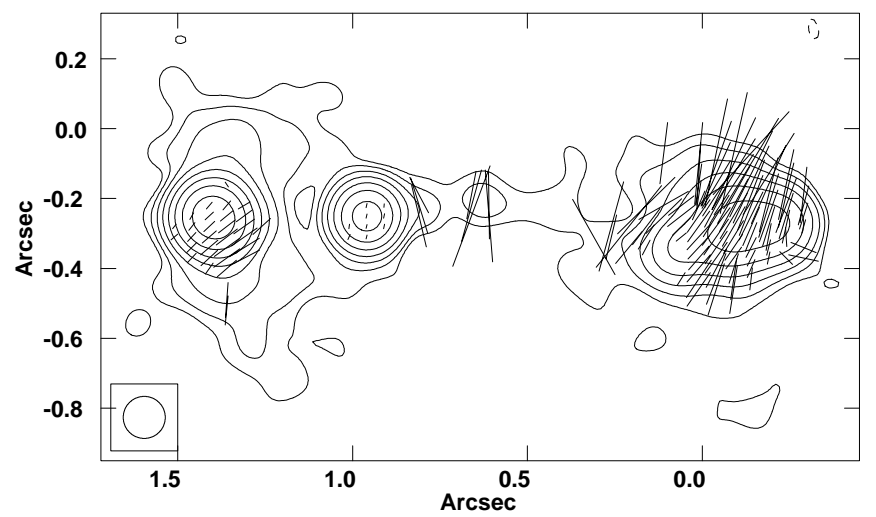

Fig. 6. Contours of the $2 \mathrm{~cm} 0.12^{\prime \prime}$ resolution image with vectors whose length are proportional to the $2 \mathrm{~cm}$ fractional polarization and the derived intrinsic polarization angle. Contours are powers of 2 times $200 \mu \mathrm{Jy}$. The image is rotated by $-42^{\circ}$.

parameters $s_{0} / b$ and $\sigma$ are degenerate so we applied the model of Burn (1966) only. For each pixel at which fractional polarizations were available at a minimum of two wavelengths, the two parameter ( $m$ and $\sigma$ ) Burn model was fitted. This fitting was done by a multi-stage direct parameter search procedure. The results of this fitting are shown in Fig. 9; data and model fits for selected positions are shown in plots at the bottom.

The behavior shown in Fig. 9 is relatively consistent over the western lobe but varies dramatically between lobes and in the core and across the eastern lobe. In the western lobe, the derived intrinsic fractional polarization is typically 10-20\% and the Faraday dispersion low, $\sim 150 \mathrm{rad} / \mathrm{m}^{2}$. This lobe is only weakly depolarized but the Burn model gives a poor fit

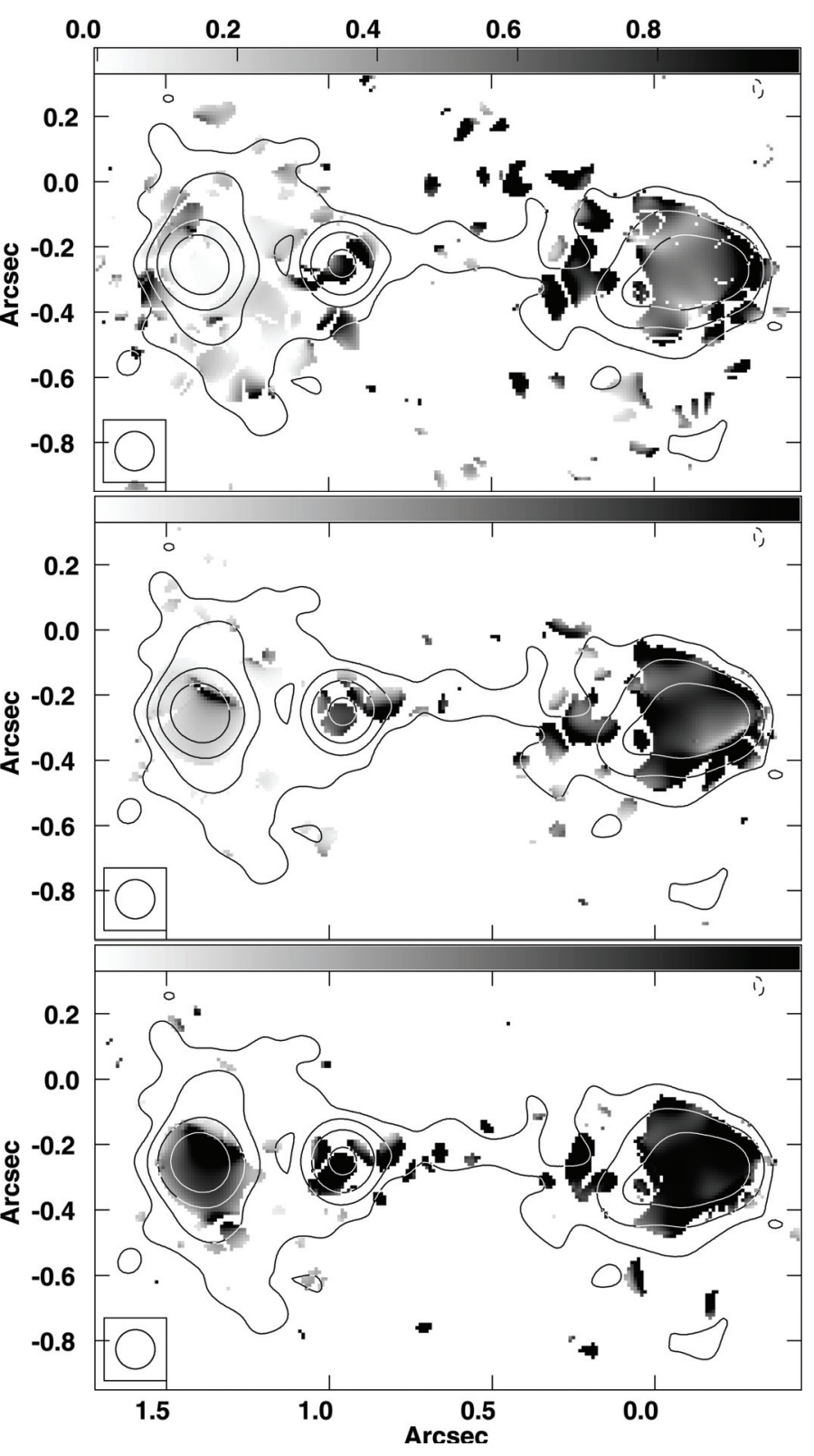

Fig. 7. Top: the gray scale shows the ratio of the fractional polarization at $6 \mathrm{~cm}$ to that at $1.3 \mathrm{~cm}$ with superposed contours of the $2 \mathrm{~cm}$ $0.12^{\prime \prime}$ resolution image. Contours are powers of 4 times $100 \mu \mathrm{Jy}$. The image is rotated by $-42^{\circ}$. Middle: like top but the ratio of 3.6 to $1.3 \mathrm{~cm}$ fractional polarizations. Bottom: like top but the ratio of 6 to $1.3 \mathrm{~cm}$ fractional polarizations.

to the fractional polarization data, especially at $3.6 \mathrm{~cm}$. The fractional polarization at the core is low, of order $1 \%$ or less at all wavelengths (see Fig. 7) hence, a low Faraday dispersion. This is consistent with the relatively low rotation measure seen in Fig. 5. The eastern lobe shows more strongly the expected effects of a Faraday screen. The intrinsic fractional polarization varies from $\sim 10 \%$ in the southern regions of the lobe and drops to below the detection threshold of a couple percent on the northern edge. The Faraday dispersion in this lobe is large everywhere, from $\sim 600 \mathrm{rad} / \mathrm{m}^{2}$ near the center to $\sim 1500 \mathrm{rad} / \mathrm{m}^{2}$ near the edges. This is to be compared to the observed rotation measures of order $-1000 \mathrm{rad} / \mathrm{m}^{2}$ detected in 


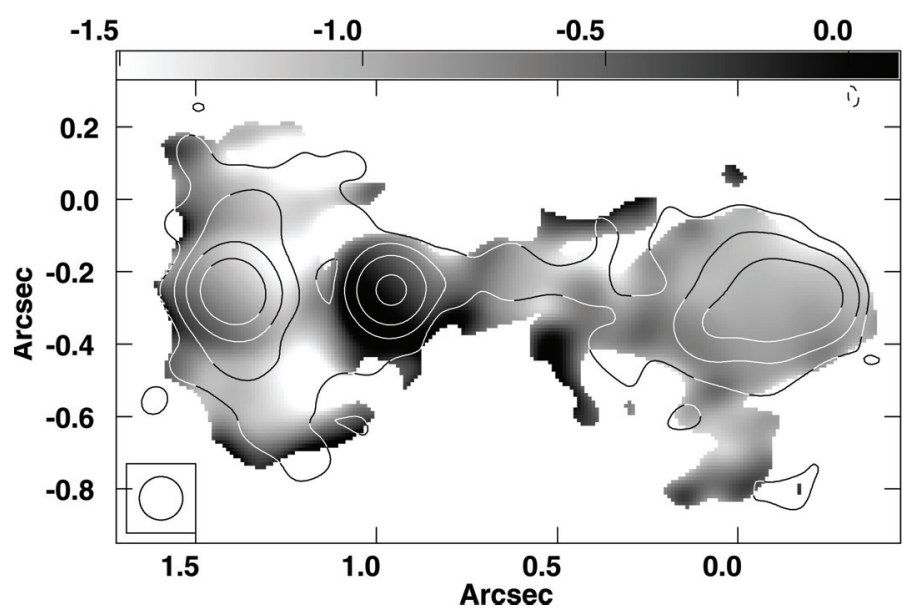

Fig. 8. The gray scale shows the spectral index at $0.12^{\prime \prime}$ resolution between 6 and $1.3 \mathrm{~cm}$ with superposed contours of the $2 \mathrm{~cm} 0.12^{\prime \prime}$ resolution image. Contours are powers of 4 times $100 \mu \mathrm{Jy}$. The image is rotated by $-42^{\circ}$.

this lobe in Fig. 5. Although this lobe is strongly depolarized at longer wavelengths, the Burn model can adequately represent the observations in contrast to the western lobe. The innermost knot in the western jet can be seen in Fig. 9 has an intrinsic degree of polarization, $m_{0}$, of $10 \%$ which is comparable to typical values in the western lobe.

\subsection{Comparison with [OIII] HST image}

A detailed comparison of the radio and the HST O[III] (Axon et al. 2000) images is given in Fig. 10. The relative astrometry of the two images is insufficient for this comparison. However, the nucleus in this source is relatively prominent so it was used to align the radio and [OIII] images. The optical emission is more extended along the axis of the radio jet than across, suggesting a relationship. The North-West radio lobe extends beyond the line emission whereas the South East lobe is closer to the core and appears to be embedded in the line emitting gas. However, since it takes some time for the shocks to become radiative, of order $10^{4}$ years (Bicknell et al. 2003), it is not clear from this figure if the North-West jet has emerged from the dense region of the ISM or the shocks associated with it have not become radiative. We note that the projected distance of this lobe is larger than the "magic" $3 \mathrm{kpc}$ radius seen by Cotton et al. (2003b) at which depolarization effects dropped in a large sample of GPS/CSS sources.

Bicknell et al. (1997) and Bicknell et al. (2003) have suggested that the [OIII] emission outside of the nuclear region arises from shocks produced from the interaction of the jet and clouds of gas in the ISM. In 3C 277.1 the [OIII] emission is not confined to the region of the jet and prominent emission regions are visible to a projected distance of $\approx 1.5 \mathrm{kpc}$. These regions may be photionized by radiation escaping from other shocks or by radiation from the nucleus. They could also be excited by shocks induced by a high-pressured lobe that is not apparent at these relatively high frequencies.
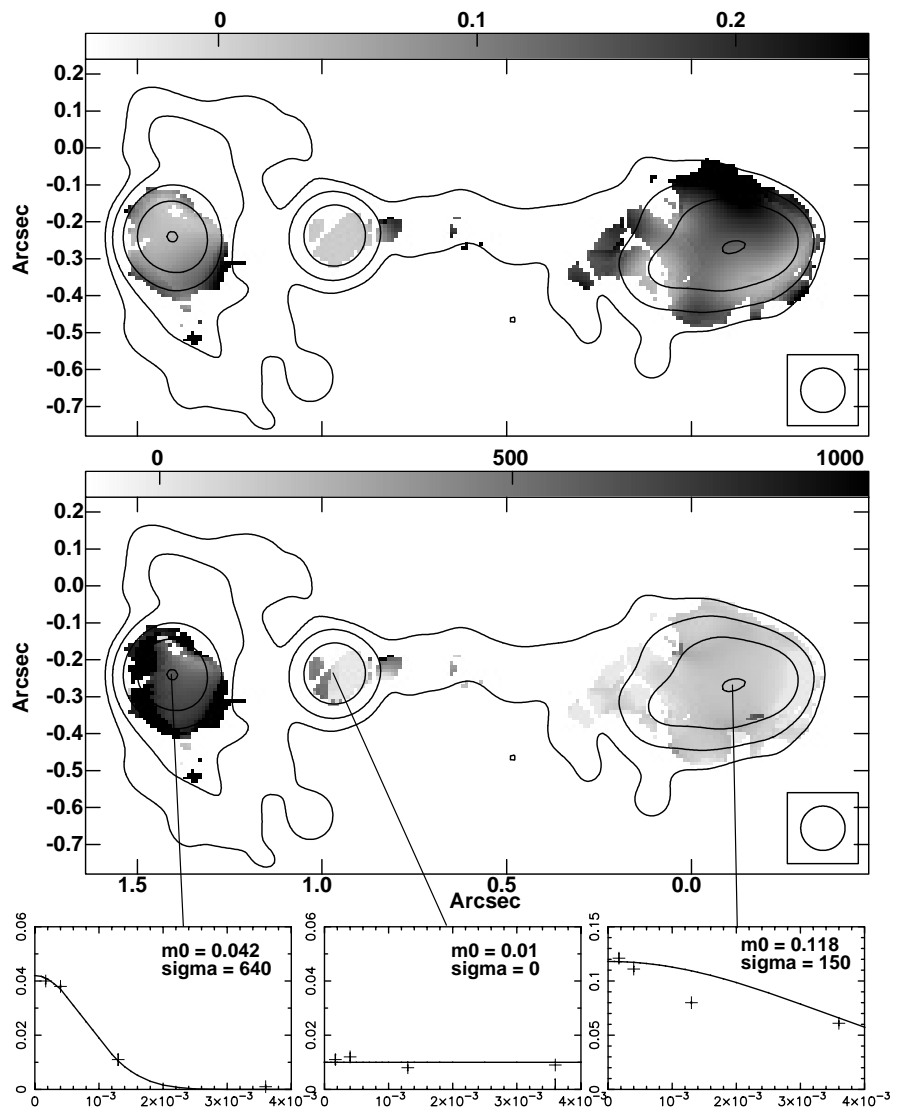

Fig. 9. Results of pixel-by-pixel fitting of Burn (1966) model at 0.12" resolution. Top: the gray scale shows the derived fractional polarization at zero wavelength with superposed contours of the $3.6 \mathrm{~cm}$ total intensity image. Contours are powers of 4 times $200 \mu \mathrm{Jy}$; the gray scale values are indicated by the wedge at the top. The image is rotated by $-42^{\circ}$. Bottom: the gray scale gives the derived Faraday dispersion $(\sigma)$ in $\mathrm{rad} / \mathrm{m}^{2}$. Shown below is the fractional polarization as a function of wavelength squared for selected locations represented as pluses (+). The solid line is the fitted model, errors equal or are less than the vertical extent of the pluses.

\subsection{Optical spectroscopy}

A study by Labiano et al. (2005) found an [OIII]/ $\mathrm{H}_{\beta}$ line ratio of $\approx 9$ in the nucleus and 5 in the extended regions. This suggests the gas in the core is photo-ionized and shocked in the extended emission.

3C 277.1 was included in the study of O'Dea et al. (2002) which found that the emission from the North-West lobe was $250-300 \mathrm{~km} \mathrm{~s}^{-1}$ higher than systemic. This velocity suggests that this is the receding side of the source. This is in disagreement with the prominence of the jet in this direction and the ratio of the arm lengths of the two sides of the source which both suggest this is the approaching side. Side-to-side differences in the ISM are likely responsible for these discrepancies.

\subsection{Spectral decomposition}

The images presented in this paper are all at wavelengths well above the long wavelength turnover in the integrated sources spectrum so do not directly bear on the source(s) of the long 


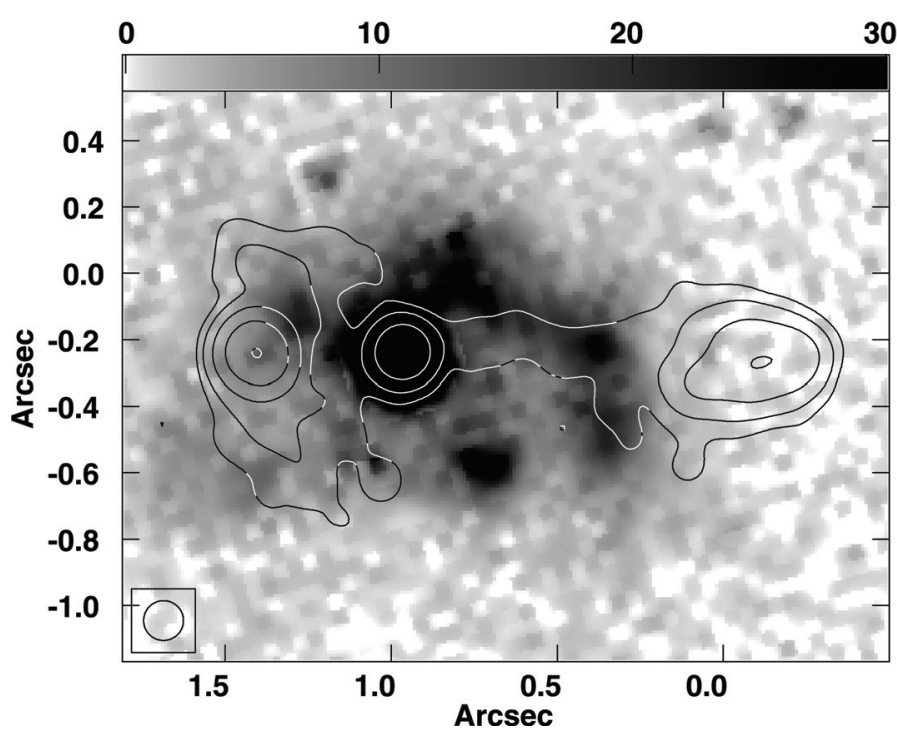

Fig. 10. Negative gray scale of the $\mathrm{O}[\mathrm{III}]$ emission image with superposed contours of the VLA $3.6 \mathrm{~cm}$ image. The bar at the top gives the values of the gray scale. Contours are powers of 4 times $200 \mu \mathrm{Jy}$. The circle in the lower left corner gives the resolution of the $3.6 \mathrm{~cm}$ image. The images are rotated by $-42^{\circ}$.

wavelength opacity. However, these short wavelength images together with integrated flux densities from the literature can be used in a fitting procedure to determine the parameters of simple models of the long wavelength opacity. Integrated flux densities of the two lobes and the core were determined from integrals in the image (AIPS IMEAN). These values as well as integrated flux densities were then used in a direct parameter search least squares procedure to determine best fit models of the components.

The component model included two opacity types, synchrotron and free-free:

$S_{v}=S_{0}\left(\frac{v}{v_{0}}\right)^{2.5} \times\left[1-\mathrm{e}^{-\left(\frac{\nu}{v_{0}}\right)^{-\alpha-2.5}}\right] \times \mathrm{e}^{-\frac{f}{v^{2}}}$

where $S_{v}$ is the flux density at frequency $v, v_{0}$ is the reference frequency (for synchrotron opacity, this is the frequency of the maximum in the spectrum), $S_{0}$ is the flux density at frequency $v_{0}, \alpha$ is the spectral index and $f$ is a free-free absorption factor. In all fittings, the East lobe was the one with the lowest frequency cutoff, either free-free or synchrotron. This result is counter-intuitive as this component is the more compact which should result in a higher synchrotron cutoff and is behind more thermal plasma which should result in a higher free-free cutoff. Since the opacity source in the western component is very weakly constrained and there appears to be little plasma in its direction, the opacity source fitted in this component was always synchrotron. The best fit model parameters are given in Table 3 and the fitted spectra shown in Figs. 11 and 12.
Table 3. Best fit spectral models.

\begin{tabular}{|c|c|c|c|c|c|}
\hline Opacity & Comp. & $\begin{array}{l}S_{0} \\
\text { Jy }\end{array}$ & $\begin{array}{r}v_{0} \\
\mathrm{GHz}\end{array}$ & $\overline{\alpha \alpha}$ & $\overline{\overline{f f}}$ \\
\hline Synch. & West & 1.278 & 1.209 & 1.044 & $\overline{0.0}$ \\
\hline “ & Core & 0.164 & 0.1 & 0.286 & 0.0 \\
\hline “ & East & 34.675 & 0.0616 & 0.9845 & 0.0 \\
\hline Free-free & West & 1.702 & 0.93497 & 1.0565 & 0.0 \\
\hline “ & Core & 0.057 & 0.1 & 0.0625 & 0.0 \\
\hline “ & East & 335. & 0.005 & 0.9565 & 0.001862 \\
\hline
\end{tabular}

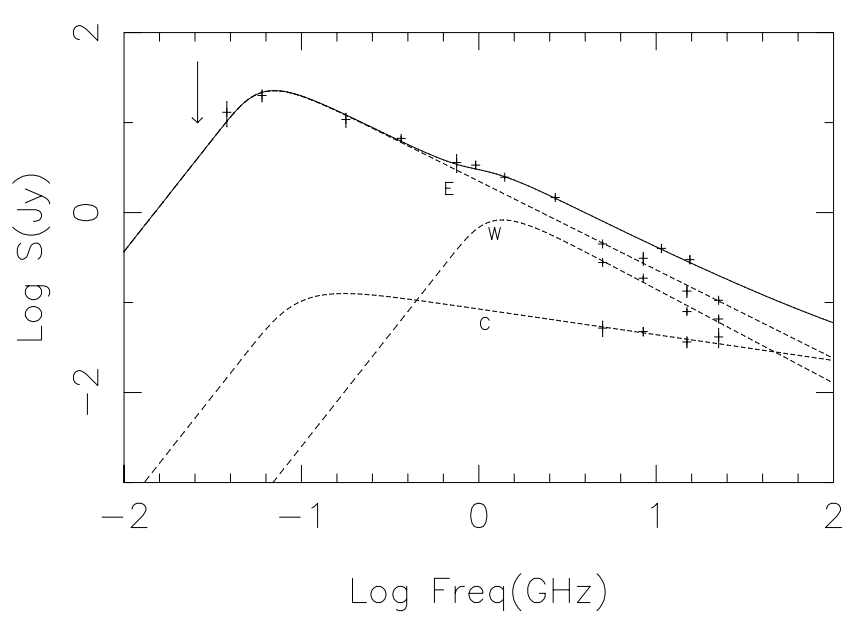

Fig. 11. Fitted spectral decomposition with only synchrotron opacity for all components. Fitted data are shown with vertical error bars. The solid line is the total model flux density and the dashed lines are individual component spectra, "C" = core, "W" = west lobe, "E" = east lobe. The west lobe has its low frequency cutoff around $1 \mathrm{GHz}$, the core has a flat spectrum and the east component has a low frequency cutoff around $0.06 \mathrm{GHz}$.

\section{Discussion}

\subsection{Comparison of radio continuum and [OIII] emission}

The regions of strong radio and [OIII] emission shown in Fig. 10 are nearly anti-correlated. A further examination of this is shown in Fig. 13 which shows the distribution of radio continuum and line emission on a pixel-by-pixel basis outside of the nuclear region. Most of the [OIII] emission arises quite far from the jet and is unlikely to be directly shock excited by the jet. Furthermore, there is no evidence in Figs. 10 and 13 that the jet is causing local enhancements of the [OIII] emission. Due to the finite time needed for the shocks to cool to the point that there is significant line emission, and the limited lifetime in this phase, [OIII] emission would not be expected everywhere along the jet, but, except for a region near the western edge of the line emitting region, the [OIII] emission appears to avoid the jet. Due to potential projection effects, even an association here is unclear; this [OIII] emission appears to be part of a large scale "spiral" apparently unassociated with the jet.

There is another region of [OIII] which is possibly associated with the jet. This is near the northern side of the eastern lobe where the northern extension of the lobe appears to be diverted further to the east, see Fig. 10. 


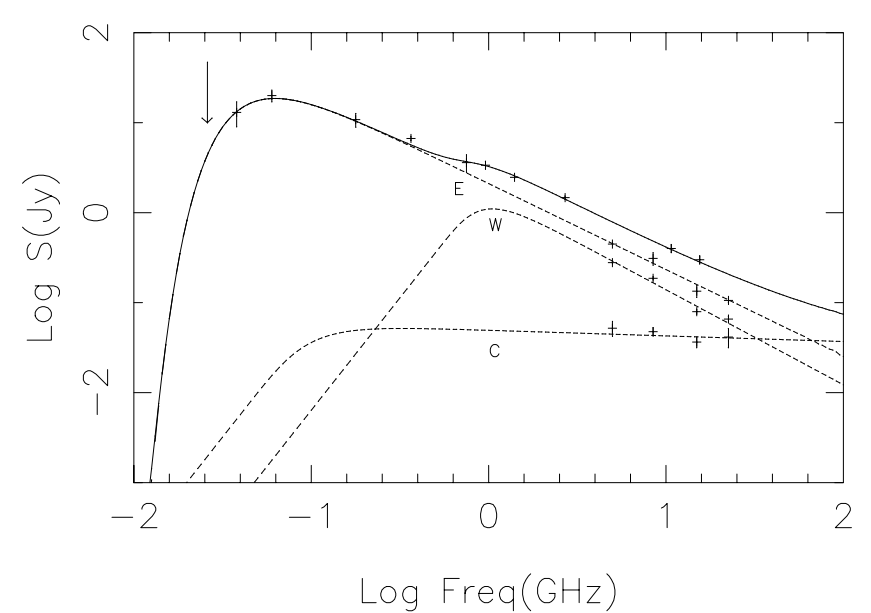

Fig. 12. Fitted spectral decomposition with only free-free opacity in the east lobe and only synchrotron opacity for the other components. Fitted data are shown with vertical error bars. The solid line is the total model flux density and the dashed lines are individual component spectra, "C"=core, "W" = west lobe, "E" = east lobe. The west lobe has its low frequency cutoff around $1 \mathrm{GHz}$, the core has a flat spectrum and the east component has a low frequency cutoff around $0.06 \mathrm{GHz}$.

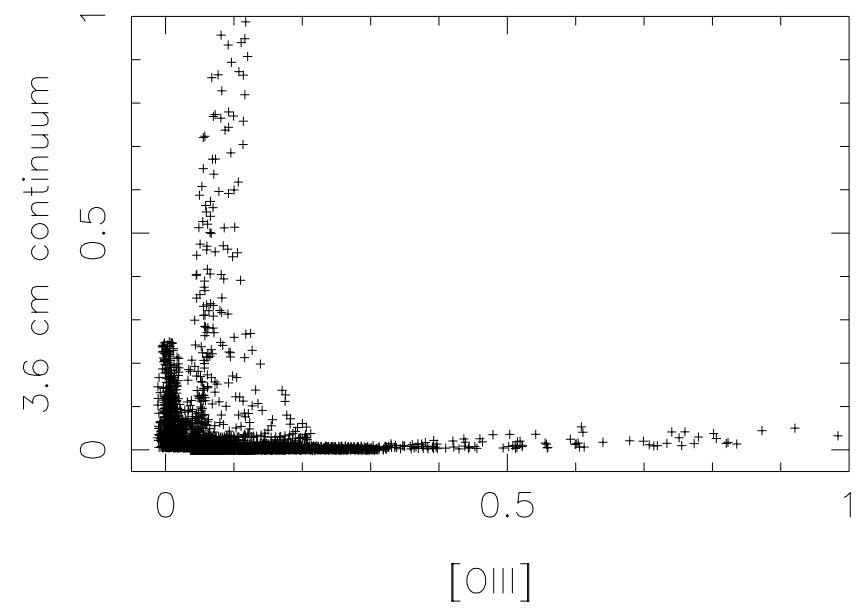

Fig. 13. Scatter plot of the radio continuum at $3.6 \mathrm{~cm}$ versus the [OIII] emission on a pixel-by-pixel basis of the images shown in Fig. 10 but excluding the nuclear region. Brightnesses are given in arbitrary units. The near anti-correlation is shown by the "L" shape of the distribution.

\subsection{Comparison of Faraday effects and [OIII] emission}

If jet-induced shocks are at a very high temperature, the cooling time until there is significant line emission can be quite long and the absence of [OIII] emission does not rule out such shocks. However, the plasma in these shocks will still affect radio emission propagating through them in the forms of freefree absorption and Faraday rotation if there is also a magnetic field. The Bicknell et al. (2003) model predicts that the shocked material would be filamentary. Large variations in the Faraday depth through different lines of sight would largely depolarize radio signals propagating through the filaments and the signals passing between the filaments would be largely unaffected. Thus, the expectation is that radio emission passing through such a shocked region would be weakly polarized, depending on the covering factor of the filaments, and that the polarized components of the signal would have relatively low Faraday rotation. This is an accurate description of what is seen in the nuclear region of the source; a low polarization at all frequencies and relatively low Faraday rotation of what remains. In the nuclear region we interpret our results as indicating a dense Faraday screen with a large, but less than unity, covering factor.

However, in the lobes the situation is different. The western lobe has relatively few apparent Faraday effects which is consistent with its apparent emergence from the line emitting region. Figure 9 illustrates this with $10-20 \%$ intrinsic polarization and low Faraday dispersion.

The first knot in the western jet has polarization properties similar to the western lobe; relatively high intrinsic polarization and low Faraday dispersion and Faraday rotation. This portion of the jet appears to have very little shocked plasma around it.

The eastern lobe is strongly depolarized with wavelength and has a relatively large overall Faraday rotation and Faraday dispersion which is well described by the Burn model. The intrinsic polarization, $m_{0}$ shown in Fig. 9 varies from $\sim 15 \%$ in the south of this lobe to less than a few percent in the north. This strong depolarization, even at relatively short wavelength is the signature we expect from viewing the source through a dense Faraday screen with large covering factor. Furthermore, the depolarization becomes stronger toward a clump of [OIII] emission discussed above. A closeup view of this region is given in Fig. 14; the left side shows the decreasing intrinsic polarization toward regions of higher [OIII] emission. Higher resolution $\left(0.06^{\prime \prime}\right) 1.3 \mathrm{~cm}$ results are given on the right showing that the southern portion of the lobe is far more polarized than the north.

The Faraday rotation over most of this lobe is relatively constant near $\sim-1000 \mathrm{rad} / \mathrm{m}^{2}$ except for the northern edge where it jumps to $\sim+2400 \mathrm{rad} / \mathrm{m}^{2}$. This suggests that the overall Faraday rotation and dispersion seen in this lobe are from a large scale distribution of magnetized plasma but there are additional and stronger Faraday effects in the north which may be due to a dense Faraday screen created by shocks from the northward expansion of the eastern lobe.

\subsection{Spectrum analysis}

The spectral decomposition shown in Figs. 11 and 12 is difficult to understand. If the basic opacity at long wavelengths is either synchrotron or free-free, the eastern component should have the higher frequency turnover as it is both the more compact and behind more thermal material. The spectral fitting has been assigning the apparent kink in the spectrum around $1 \mathrm{GHz}$ as the turnover of the western lobe. An alternate interpretation is that this feature is due to the aging of the emitting relativistic electrons. After subtracting the core from the integrated spectrum, it is well fitted by a continuous injection model with a break frequency at $4.1 \mathrm{GHz}$ and a possible turn-over at about $50 \mathrm{MHz}$. Assuming an equipartition magnetic field of $\sim 1 \mathrm{mG}$, this gives an age $\sim 4 \times 10^{4} \mathrm{yr}$ and average projected velocities of the heads of the lobes of $0.15 \mathrm{c}$ and $0.06 \mathrm{c}$ for the western and eastern lobes. 

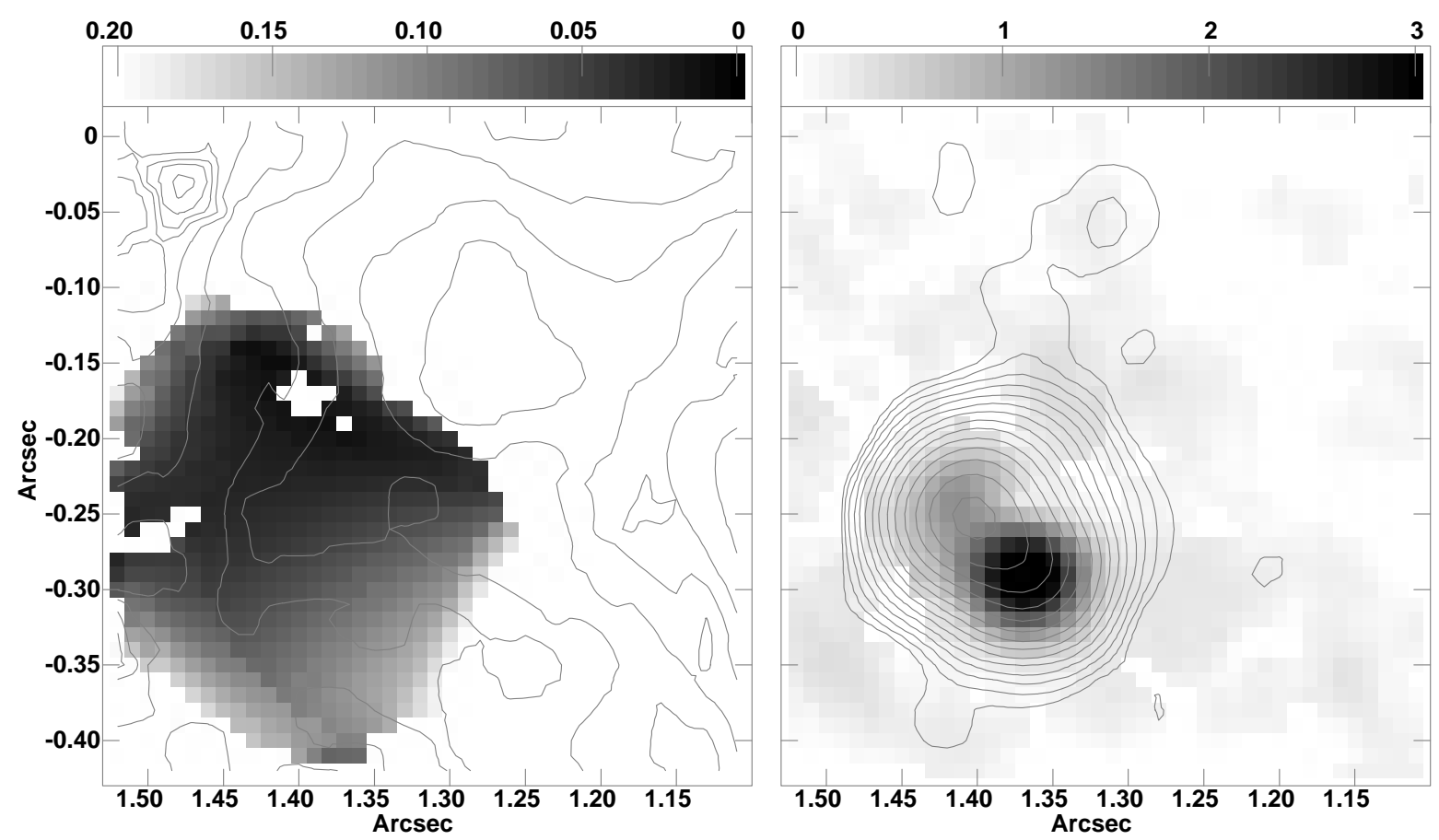

Fig. 14. Left: closeup of east lobe of 3C 277.1 with the intrinsic polarization ( $m_{0}$ from Burn model fit to $0.12^{\prime \prime}$ resolution data) in negative grayscale and the HST [OIII] emission as contours. The decreasing polarization fraction toward the region of [OIII] emission is readily apparent. The scale of the intrinsic polarization is given by the wedge at the top. The [OIII] contour are factors of $\sqrt{2}$ and are arbitrary units The image is rotated by $-42^{\circ}$. Right: same area as left but showing $1.3 \mathrm{~cm} 0.06^{\prime \prime}$ resolution bias corrected polarized intensity as grayscale and total intensity as contours. The gray-scale is given in mJy by the wedge at the top; contours are are factors of $\sqrt{2}$ times $0.5 \mathrm{mJy}$.

\subsection{Jet orientation}

The evidence from the radio source largely points in the direction of the western jet being the approaching one, these are jet sidedness and arm length ratio. The difference in morphology does suggest an intrinsic difference in the ISM encountered by the two jets. The eastern jet appears to be broadened near the head whereas the western one is not. The much stronger Faraday effects on the eastern side of the source suggest that this is the receding one of the jet (Laing-Garrington effect). The optical evidence of O'Dea et al. (2002) is that [OIII] emission in the area of the Western lobe was $250-300 \mathrm{~km} \mathrm{~s}^{-1}$ higher than systemic. If this gas is physically associated with the jet, this would suggest that this jet were the receding one. From the arguments given above, it is unclear if this gas is associated with the jet. If the emitting cloud is associated with the jet but were on the far side of it, the cloud could be accelerated away from us.

\subsection{Long wavelength opacity}

It is not possible on the basis of material presented here to determine the origin of the long wavelength opacity in 3C 277.1. If the opacity is free-free in shocked material surrounding the jet, then this material has not cooled sufficiently to begin emitting in the [OIII] line. There is thermal plasma in front of the eastern lobe as evidenced by the Faraday effects seen in the radio emission. However, free-free opacity and Faraday rotation depend on different powers of the density, as well as the magnetic field dependence of Faraday effects, so it is not possible to convert from Faraday effects to free-free opacity. There is no evidence suggesting that free-free absorption is significant in the western lobe.

\section{Conclusions}

There appears to be no detailed association of [OIII] emission with the radio jet. Much of the optical emission is sufficiently far from the jet that it is unlikely to arise directly from shocks induced by the passage of the jet through the ISM. The finite cooling time for the shock to become radiative may make the association of shocks and jets using only the optical line emission more difficult. Significant Faraday effects are seen in the radio emission from the jet but there is also little evidence that the Faraday screen comes from the immediate vicinity of the jet and could be due to passage of the radiation through the NLR. The western lobe appears to have emerged from the region of dense plasma but the eastern lobe is behind a considerable amount of magnetized plasma. In addition, the northern edge of the east lobe is strongly depolarized, even at relatively short wavelengths and with large variations in the Faraday rotation. We interpret these effects as possibly being due to an especially dense plasma screen arising from jet-induced shocks. Further supporting this interpretation is the presence of higher [OIII] emission in this area. Thus, overall, the jet seems to have little interaction with the ISM except possibly in a limited region of the eastern lobe. However, we cannot rule out the possibility that some of the [OIII] emission not clearly associated with the radio emission is shock excited by an extended, very steep spectrum radio lobe not detected in the current data. 
Interpretation of the integrated radio source spectrum in terms of a continuous injection model gives a spectral age of $\sim 4 \times 10^{4} \mathrm{yr}$ and projected velocities of the heads of the lobes of $0.15 \mathrm{c}$ and $0.06 \mathrm{c}$ for the western and eastern lobes.

The source of the opacity causing the long wavelength turnover in the radio spectrum remains uncertain. Near the nucleus of the source, the low fractional polarization and low Faraday rotation suggest that the emission has passed through a dense Faraday screen with a high covering factor. Emission within a projected distance of the nucleus of $3 \mathrm{kpc}$ show strong Faraday effects whereas outside of this distance show much weaker Faraday effects, in agreement with Cotton et al. (2003b) and Fanti et al. (2004).

Acknowledgements. The authors would like to thank A. Capetti for providing the HST O[III] image.

\section{References}

Axon, D. J., Capetti, A., Fanti, R., et al. 2000, AJ, 120, 2284

Bicknell, G. V., Dopita, M. A., \& O’Dea, C. P. 1997, AJ, 485, 112
Bicknell, G. V., Saxton, C. J., \& Sutherland, R. S. 2003, PASA, 20, 102

Burn, B. J. 1966, MNRAS, 133, 67

Cotton, W. D., Dallacasa, D., Fanti, C., et al. 2003a, A\&A, 406, 43

Cotton, W. D., Dallacasa, D., Fanti, C., et al. 2003b, PASA, 20, 12

Fanti, C., Fanti, R., Dallacasa, D., et al. 1995, A\&A, 302, 317

Fanti, C., Branchesi, M., Cotton, W. D., et al. 2004, A\&A, 427, 465

Gelderman, R., \& Whittle, M. 1994, ApJS, 91, 491

Jeyakumar, S., Wiita, P. J., Saikia, D. J., \& Hooda, J. S. 2005, A\&A, 432,823

Kapahi, V. K. 1981, A\&AS, 43, 381

Labiano, A., O’Dea, C. P., Gelderman, R., et al. 2005, A\&A, 436, 493

Lüdke, E., Garrington, S. T., Spencer, R. E., et al. 1998, MNRAS, 299, 467

O'Dea, C. P. 1998, PASP, 110, 493

O'Dea, C. P., de Vries, W. H., Koekemoer, A. M., et al. 2002, AJ, 123, 2333

Peacock, J. A., \& Wall, J. V. 1982, MNRAS, 198, 843

Readhead, A. C. S., Taylor, G. B., Pearson, T. J., \& Wilkinson, P. N. 1996, ApJ, 460, 634

Saikia, D. J., \& Gupta, N. 2003, A\&A, 405, 499

Tribble, P. C. 1991, MNRAS, 250, 726 